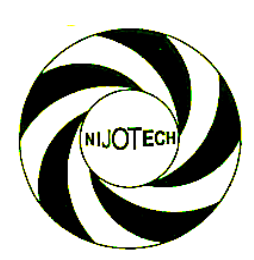

Nigerian Journal of Technology (NIJOTECH)

Vol. 38, No. 2, April 2019, pp. 364 - 375

Copyright@ Faculty of Engineering, University of Nigeria, Nsukka,

Print ISSN: 0331-8443, Electronic ISSN: 2467-8821 www.nijotech.com

http://dx.doi.org/10.4314/njt.v38i2.13

\title{
MULTI-RESPONSE OPTIMISATION OF MACHINING PARAMETERS IN TURNING AISI 304L USING DIFFERENT OIL-BASED CUTTING FLUIDS
}

\author{
C. K. Agu1, S. A. Lawal2, *, M. S. Abolarin3, J. B. Agboola4, \\ J. Abutu ${ }^{5}$ and E. I. Awode 6

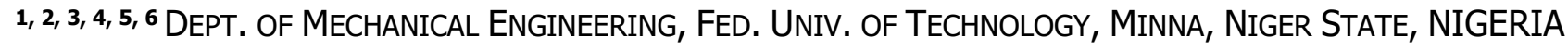 \\ E-mail addresses: ${ }^{1}$ kannyagu2@yahoo.com; 2 lawalsunday@futminna.edu.ng; \\ 3 msabolarin@futminna.edu.ng; ${ }^{4}$ joeagboola@gmail.com; 5 joe4abutu@gmail.com; \\ 6 greatawode@gmail.com
}

\begin{abstract}
Properties of melon seed and beniseed oils which are considered as "environmentally" friendly oils were investigated and the performance evaluation of the formulated beniseed and melon seed oilbased cutting fluids were carried out. American Iron and Steel Institute (AISI) 304L alloy steel was used as workpiece and tungsten carbide as cutting tool, while commercial mineral oil- based cutting fluid was used as a control experiment. The viscosities of the melon seed oil and beniseed oil- based cutting fluids were $1.53 \mathrm{~mm}^{2 / s}$ and $0.86 \mathrm{~mm}^{2 / s}$, while their $\mathrm{pH}$ values were 8.2 and 8.7 respectively. The optimal multi-response turning parameters was achieved using cutting speed of $159 \mathrm{rev} / \mathrm{min}$ (level 3), feed rate of $0.9 \mathrm{~mm} / \mathrm{rev}$ (level 3), depth of cut of $1 \mathrm{~mm}$ (level 2) and type of cutting fluid of $1.53 \mathrm{~mm} / \mathrm{s}$ (level 3). The ANOVA results show that feed rate has the most significant effect on the surface roughness (92.93\%) and cutting temperature (27.51\%).
\end{abstract}

Keywords: Cutting fluids; Surface roughness; Temperature; Cutting tool.

\section{INTRODUCTION}

Metal cutting process form the foundation of the engineering industry and is involved either indirectly or directly in the manufacture of nearly every product of our modern civilization [1]. Metal cutting is associated with high temperatures which strongly affect the accuracy of the machining operation [2]. The high forces in machining create a considerable amount of heat near the cutting edge. Most of this heat is generated within the shearing process and some heat is created by friction between the tool and workpiece. Much of the generated heat is carried off in the chips, while the remainder stays in the tool and workpiece, creating a large amount of thermal stress and softening of the cutting tool. This causes wear on the cutting edge of the tool thereby increasing the surface roughness of the sample. To minimise the adverse effect of the high temperatures, cutting fluid (coolant) is often used to bathe the tool and workpiece to reduce much of the heat and limit damage to the tool and workpiece. The three major cutting parameters in turning operation are the cutting speed, feed rate and depth of cut, these parameters need to be properly determined to achieve high performance result [3]. These cutting parameters are reflected on surface roughness, surface texture and dimensional deviations of the product [4]. Most of these cutting fluids in use presently are of mineral oil origin. However, they are environmentally unfriendly, costly, potentially toxic and with health challenges [5]. A shift to dry cutting has not completely solved the problem [6]. Hill [7] investigated the use of fats and oils as oleochemical raw materials and noted that there is tremendous increase of approximately $3 \%$ yearly in the production of oils and fats from plants and animals. It was predicted that this trend of oils and fats production from plant and animals will continue in the medium and long terms. 
Hwang and Lee [8] investigated minimum quantity lubrication and wet turning process of AISI 1045 workpiece material with the objective of determining the experimental model to predict the cutting force and surface roughness, with a view to selecting the optimal cutting parameters and to analysing the effect of cutting parameters on machinability. Result of the experiment showed that cutting speed and depth of cut had opposite effects on cutting force and surface roughness. Also, Lawal et al [9] evaluated the influence of cutting fluids on flank wear during turning of AISI 4340 alloy steel with coated carbide inserts. The performances of three types of cutting fluids were compared using Taguchi method. The effect of palm kernel oil- based emulsion cutting fluid, cotton seed oil- based emulsion cutting fluid and mineral oil- based emulsion cutting fluids on flank wear during turning of AISI 4340 alloy steel with coated carbide inserts were investigated. The result showed the optimal cutting parameters for the flank wear using signal to noise ratio as: $160 \mathrm{~m} / \mathrm{min}$ of cutting speed, $0.18 \mathrm{~mm} / \mathrm{rev}$ of feed, $1.75 \mathrm{~mm}$ depth of cut and $2.97 \mathrm{~mm}^{2} / \mathrm{s}$ flow rate for palm kernel oil-based cutting fluid. Analysis of variance showed cutting speed (85.36\%) and feed rate $(4.81 \%)$ as significant factors that affects flank wear, while depth of cut $(2.5 \%)$ and cutting fluid $(1.8 \%)$ were the insignificant factors. Bajic and majce [10] also investigated the influence of cutting parameters on the surface roughness, during the longitudinal turning process. It was reported that the increase of the cutting speed in a certain interval affected the improvement of the surface roughness. The feed has the greatest impact on the surface roughness such that the more it decreased, the more it improved the surface roughness and the increase of depth of cut improved the surface roughness as well, which can be taken as an advantage for the improvement in productivity.

Onuoha [11] also investigated the suitability of vegetable-based oils in orthogonal machining of 1330 carbon steel using Taguchi method. Machining of medium carbon steel AISI 1330 carbon steel was carried out with high speed steel (HSS) cutting tool using the formulated cutting fluids to ascertain their performance as compared with the conventional mineral oil- based cutting fluid. The experimental results showed that feed rate had the most significant effect on the surface roughness while cutting speed had the most significant effect $(66.54 \%)$ on cutting temperature. Yu et al [12] also formulated a cutting fluid using castor acid, castor oil and soybean oil. The experimental results indicated that the cutting fluids had good cooling, cleaning, anti-rust, anti-corrosive and lubricating properties, as well as having stable and reliable quality, long service life, easily available raw materials and low production cost.

Yivo et al [13] reported that grey relational analysis (GRA) optimisation procedure is a multi-response optimization technique which involves combining all performance characteristics into a particular value which can be utilised as the single characteristic in optimisation problems. Therefore, in this study, a coated carbide tool was used to evaluate the performance of melon seed and beniseed oil- based cutting fluids, compared to mineral oil- based cutting fluid, when machining AISI 304L austenitic stainless steel alloy workpiece.

\section{MATERIALS AND METHODS}

\subsection{Materials}

\subsubsection{Cutting Fluid Formulation}

The materials used in this study for the formulation of the cutting fluid include melon seed oil, beniseed oil and mineral soluble oil (MobilMet 424). Other additives used include emulsifier ( $0.5 \mathrm{M}$ sodium lauryl sulphate + sodium tripolyphospate + sulphonic acid + calcium carbonate in 5litres of water), banana plant juice (anticorrosion agent) and biocide

\subsubsection{Workpiece, cutting tool and equipment}

The materials used for the machining processes include TNMG 1604 tungsten carbide insert cutting tool, 4-jaw lathe and an AISI 304L stainless steel.

\subsection{Methods}

\subsubsection{Physicochemical Properties and Fatty Acid Composition}

The physicochemical properties of the melon seed and beniseed oils were analyzed using standard testing procedure such ASTM D445, ASTM D93 and ASTM D4052 while determination of fatty acid composition was conducted using a gas chromatograph [Mass spectrometer (GC-MS) instrument GC-MS-QP2010 Shimadzu system, Japan] with the following conditions employed: Column over temperature of $70.0^{\circ} \mathrm{C}$; injection temperature of $250.0^{\circ} \mathrm{C}$; Column flow was $1.80 \mathrm{~mL} / \mathrm{min}$ with total flow of $40.8 \mathrm{~mL} / \mathrm{min}$ at linear velocity of $49.2 \mathrm{~cm} / \mathrm{sec}$ and pressure of $116.9 \mathrm{kpa}$.

\subsubsection{Formulation of Emulsion Cutting Fluid}

The preparation of cutting fluid was based on the method adopted by Muniz et al [14]. This procedure 
involved controlled addition of additives to the oil using a magnetic stirrer (Hotplate 79-1). The percentage ratios of additives used were $9.35 \%$ emulsifier, $0.97 \%$ biocide, $10.61 \%$ anticorrosive agent, $0.64 \%$ antioxidant [11]. However, the formulation of mineral based cutting fluid involved mixing the soluble oil (concentrate) with water at the ratio of $1: 9$.

\subsubsection{Characterization of Formulated Cutting Fluids}

The formulated cutting fluids were characterized to determine the $\mathrm{pH}$ value, viscosity, corrosion level and stability. The $\mathrm{pH}$ values were measured with $\mathrm{pH}$ meter while viscosity was determined using ASTM D445 measurement procedure. The corrosion level was determined using the test method adopted by [15], while the stability was evaluated using a visual transparency within a period of 24 hours at room temperature as to the separation of water and oil in a graduated $1000 \mathrm{ml}$ test tubes.

\subsubsection{Design of Experiment}

DOE was carried out in accordance with full factorial $\mathrm{L}_{81}(3)^{4}$ design technique using Minitab 17 statistical software. The factor levels of input variables are shown in Table 1 while the experimental design layout is shown in Table 2.

Table 1: Factor Levels of Machining Variables

\begin{tabular}{|c|c|c|c|c|}
\hline Factor & Unit & $\begin{array}{l}\text { Level } 1 \\
(-)\end{array}$ & $\begin{array}{c}\text { Level } 2 \\
(0)\end{array}$ & $\begin{array}{c}\text { Level } 3 \\
(+)\end{array}$ \\
\hline Cutting & $\mathrm{rev} / \mathrm{min}$ & 93 & 126 & 159 \\
\hline \multicolumn{5}{|l|}{ Speed } \\
\hline Feed Rate & $\mathrm{mm} / \mathrm{rev}$ & 0.5 & 0.7 & 0.9 \\
\hline Depth of Cut & $\mathrm{Mm}$ & 0.8 & 1.0 & 1.2 \\
\hline $\begin{array}{l}\text { Types of } \\
\text { Cutting Fluids }\end{array}$ & $\mathrm{mm}^{2} / \mathrm{s}$ & MSO & BSO & CMO \\
\hline
\end{tabular}

\subsubsection{Machining Process}

Turning operation was conducted on a 4-jaw conventional lathe (MITCHELL of Keighley, variable spindle speed: $30-400 \mathrm{rpm}$ and $5 \mathrm{Hp}$ rated power). A triangular tungsten carbide tool inserts (TNMG 1604, Canela Tools) was used with a new cutting edge for each experiment. The investigation of surface roughness and cutting temperature were conducted on stainless steel (AISI 304L) round bars of $80 \mathrm{~mm}$ diameter by $320 \mathrm{~mm}$ length to achieve a ratio of

diameter to length of $1: 4$ which ensures rigidity and eliminates flexing during the turning operation [16]. Each test specimen was mounted on the lathe between the chuck and the live centre to ensure greater clamping force during turning. A thin outer surface of each specimen was machined off before the start of the experiments for uniformity. The workpiece was afterward turned at different depths, turning speeds and feed rates as specified in Table 2 .

Table 2: Full Factorial $\mathrm{L}_{81}(3)^{4}$ Experimental Design Layout

\begin{tabular}{|c|c|c|c|c|}
\hline $\begin{array}{l}\text { Run } \\
\text { Order }\end{array}$ & $\begin{array}{l}\text { Cutting } \\
\text { Speed }\end{array}$ & $\begin{array}{l}\text { Feed } \\
\text { Rate }\end{array}$ & $\begin{array}{l}\text { Depth } \\
\text { of Cut }\end{array}$ & $\begin{array}{l}\text { Type of } \\
\text { Cutting } \\
\text { Fluids }\end{array}$ \\
\hline 1 & 93 & 0.5 & 0.8 & MSO \\
\hline 2 & 93 & 0.9 & 1.2 & CMO \\
\hline 3 & 159 & 0.9 & 0.8 & MSO \\
\hline 4 & 159 & 0.7 & 0.8 & BSO \\
\hline 5 & 159 & 0.9 & 1 & CMO \\
\hline 6 & 126 & 0.9 & 1.2 & CMO \\
\hline 7 & 126 & 0.7 & 0.8 & CMO \\
\hline 8 & 126 & 0.9 & 0.8 & CMO \\
\hline 9 & 126 & 0.7 & 0.8 & MSO \\
\hline 10 & 159 & 0.5 & 1.2 & CMO \\
\hline 11 & 159 & 0.5 & 1 & CMO \\
\hline 12 & 159 & 0.5 & 0.8 & BSO \\
\hline 13 & 93 & 0.9 & 1 & MSO \\
\hline 14 & 126 & 0.7 & 1.2 & CMO \\
\hline 15 & 93 & 0.7 & 0.8 & MSO \\
\hline 16 & 93 & 0.7 & 1.2 & BSO \\
\hline 17 & 93 & 0.7 & 1.2 & MSO \\
\hline 18 & 93 & 0.5 & 1.2 & BSO \\
\hline 19 & 93 & 0.5 & 0.8 & BSO \\
\hline 20 & 126 & 0.9 & 0.8 & MSO \\
\hline 21 & 159 & 0.7 & 1.2 & BSO \\
\hline 22 & 159 & 0.9 & 1 & MSO \\
\hline 23 & 126 & 0.9 & 1 & MSO \\
\hline 24 & 93 & 0.7 & 1.2 & CMO \\
\hline 25 & 159 & 0.9 & 1.2 & BSO \\
\hline 26 & 93 & 0.7 & 0.8 & $\mathrm{CMO}$ \\
\hline 27 & 159 & 0.9 & 1.2 & MSO \\
\hline 28 & 126 & 0.5 & 1.2 & BSO \\
\hline 29 & 159 & 0.7 & 1 & MSO \\
\hline 30 & 159 & 0.9 & 0.8 & BSO \\
\hline 31 & 126 & 0.5 & 1.2 & MSO \\
\hline 32 & 159 & 0.7 & 1.2 & CMO \\
\hline 33 & 159 & 0.7 & 1 & CMO \\
\hline 34 & 93 & 0.9 & 1 & CMO \\
\hline 35 & 126 & 0.5 & 1 & MSO \\
\hline 36 & 126 & 0.7 & 1 & MSO \\
\hline 37 & 159 & 0.5 & 0.8 & $\mathrm{CMO}$ \\
\hline 38 & 159 & 0.9 & 1.2 & CMO \\
\hline 39 & 93 & 0.9 & 0.8 & CMO \\
\hline 40 & 159 & 0.5 & 1 & BSO \\
\hline 41 & 126 & 0.7 & 0.8 & BSO \\
\hline 42 & 126 & 0.9 & 1.2 & MSO \\
\hline 43 & 93 & 0.7 & 1 & BSO \\
\hline 44 & 93 & 0.9 & 0.8 & BSO \\
\hline
\end{tabular}




\begin{tabular}{|c|c|c|c|c|}
\hline $\begin{array}{l}\text { Run } \\
\text { Order }\end{array}$ & $\begin{array}{l}\text { Cutting } \\
\text { Speed }\end{array}$ & $\begin{array}{l}\text { Feed } \\
\text { Rate }\end{array}$ & $\begin{array}{l}\text { Depth } \\
\text { of Cut }\end{array}$ & $\begin{array}{l}\text { Type of } \\
\text { Cutting } \\
\text { Fluids }\end{array}$ \\
\hline 45 & 93 & 0.9 & 1.2 & BSO \\
\hline 46 & 159 & 0.7 & 0.8 & $\mathrm{CMO}$ \\
\hline 47 & 126 & 0.5 & 1.2 & $\mathrm{CMO}$ \\
\hline 48 & 159 & 0.5 & 1.2 & BSO \\
\hline 49 & 93 & 0.5 & 1 & BSO \\
\hline 50 & 93 & 0.7 & 0.8 & BSO \\
\hline 51 & 126 & 0.9 & 1.2 & BSO \\
\hline 52 & 126 & 0.9 & 0.8 & BSO \\
\hline 53 & 126 & 0.9 & 1 & BSO \\
\hline 54 & 159 & 0.7 & 1 & BSO \\
\hline 55 & 126 & 0.5 & 1 & BSO \\
\hline 56 & 93 & 0.9 & 1 & BSO \\
\hline 57 & 159 & 0.9 & 1 & BSO \\
\hline 58 & 126 & 0.7 & 1.2 & MSO \\
\hline 59 & 93 & 0.5 & 0.8 & $\mathrm{CMO}$ \\
\hline 60 & 93 & 0.5 & 1.2 & $\mathrm{CMO}$ \\
\hline 61 & 126 & 0.9 & 1 & $\mathrm{CMO}$ \\
\hline 62 & 93 & 0.5 & 1 & MSO \\
\hline 63 & 126 & 0.7 & 1 & BSO \\
\hline 64 & 93 & 0.7 & 1 & $\mathrm{CMO}$ \\
\hline 65 & 126 & 0.7 & 1.2 & BSO \\
\hline 66 & 93 & 0.7 & 1 & MSO \\
\hline 67 & 159 & 0.5 & 1.2 & MSO \\
\hline 68 & 159 & 0.7 & 0.8 & MSO \\
\hline 69 & 159 & 0.5 & 1 & MSO \\
\hline 70 & 159 & 0.7 & 1.2 & MSO \\
\hline 71 & 159 & 0.5 & 0.8 & MSO \\
\hline 72 & 126 & 0.5 & 1 & $\mathrm{CMO}$ \\
\hline 73 & 126 & 0.5 & 0.8 & MSO \\
\hline 74 & 126 & 0.5 & 0.8 & $\mathrm{CMO}$ \\
\hline 75 & 126 & 0.7 & 1 & $\mathrm{CMO}$ \\
\hline 76 & 126 & 0.5 & 0.8 & BSO \\
\hline 77 & 159 & 0.9 & 0.8 & $\mathrm{CMO}$ \\
\hline 78 & 93 & 0.9 & 1.2 & MSO \\
\hline 79 & 93 & 0.9 & 0.8 & MSO \\
\hline 80 & 93 & 0.5 & 1.2 & MSO \\
\hline 81 & 93 & 0.5 & 1 & CMO \\
\hline
\end{tabular}

\subsubsection{Surface Roughness Measurement}

Surface integrity of each machined portion of the workpiece were measured using Surface Roughness Tester (Model: STR-6210S, GuangZhouLandtek) at three locations around the circumference of the round bar workpiece and the average of these reading recorded for each experiment.

\subsubsection{Cutting Temperature Measurement}

The highest cutting temperature during each experimental run was measured using a Contact Digital Thermometer (Model: TP300, range: -50 to $300^{\circ} \mathrm{C}$ ). Each experimental run was timed for a period of 10 minutes using Casio G-Shock digital stop watch.

\section{RESULTS AND DISCUSSIONS}

\subsection{Physicochemical Characterization}

Nigerian Journal of Technology,
The results of the physicochemical properties of melon seed oil (MSO) and beniseed oil (BSO) are presented in Table 3.

From the results in Table 3, it can be confirmed that the properties of melon and beniseed oils can be used for cutting fluids as the values are within the range, however with addition of additives and this is in agreement with the earlier report [17]. The low density exhibited by both oils indicates that they are lighter than water while the low cloud and pour points of oils signifies a better pumping property at relatively low temperatures. The flash points of both oils (melon and beniseed) are $315^{\circ} \mathrm{C}$ and $285^{\circ} \mathrm{C}$ respectively, which make them safe for use as lubricants while the $\mathrm{pH}$ values of the melon and beniseed oils are 3.62 and 5.28 respectively, which show that both are acidic. The acidity of oil provides a reference point for monitoring oil condition during use; as an increase in acidity during use indicates the accumulation of oxidation products in the oil [18].

Table 3: Physicochemical Properties of Melon Seed and Beniseed Oils

\begin{tabular}{clcc}
\hline S/N & Parameter & $\begin{array}{c}\text { Value (melon } \\
\text { seed) }\end{array}$ & $\begin{array}{c}\text { Value } \\
\text { (beniseed) }\end{array}$ \\
\hline 1 & Specific Gravity & 0.913 & 0.902 \\
2 & $\begin{array}{l}\text { Acid Value mg } \\
\text { KOH/g }\end{array}$ & 9.39 & 3.08 \\
& Free Fatty Acid mg & 18.79 & 6.17 \\
3 & KOH/g & & \\
& Saponification Value & 189.34 & 217.38 \\
4 & mg/g & & \\
& Viscosity mm² $\mathrm{s}^{-1} @$ & 9.38 & 11.96 \\
5 & 40 ${ }^{\circ} \mathrm{C}$ & 82.90 & 59.17 \\
6 & lodine Value g/100g & 3.87 & 2.13 \\
7 & Moisture Content \% & 3.62 & 5.28 \\
8 & pH & 315 & 285 \\
9 & Flash Point ${ }^{\circ} \mathrm{C}$ & -8 & -6 \\
10 & Cloud Point ${ }^{\circ} \mathrm{C}$ & -3 & -3 \\
11 & Pour Point ${ }^{\circ} \mathrm{C}$ & & \\
\hline
\end{tabular}

\subsection{Fatty Acid Composition}

The results of gas chromatograph -mass spectrometer (GC-MS) analysis of the melon seed and beniseed oils are presented in Fig. 1 and 2. Interpretation of mass spectrum GC-MS was done using the database of National Institute of Standard and Technology (NIST), having more than 62,000 patterns in the Library that is built into the GC-MS machine.

Tables 4 shows the fatty acid composition results of MSO and BSO. From the results, oleic acid (monounsaturated acid) has the highest values of $54.08 \%$ and $48.11 \%$ respectively for both MSO and BSO. Hence, the two oils can be classified as unsaturated. 


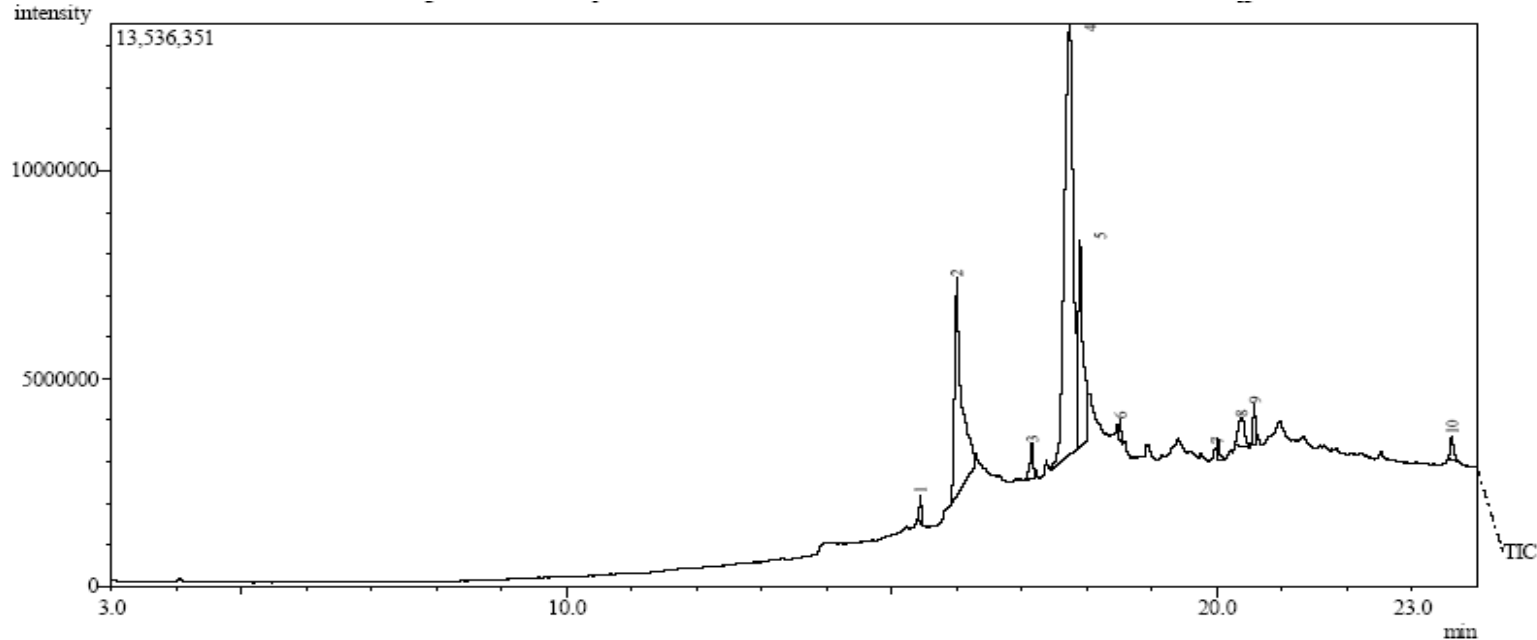

Fig. 1: GC-MS Chromatogram of MSO

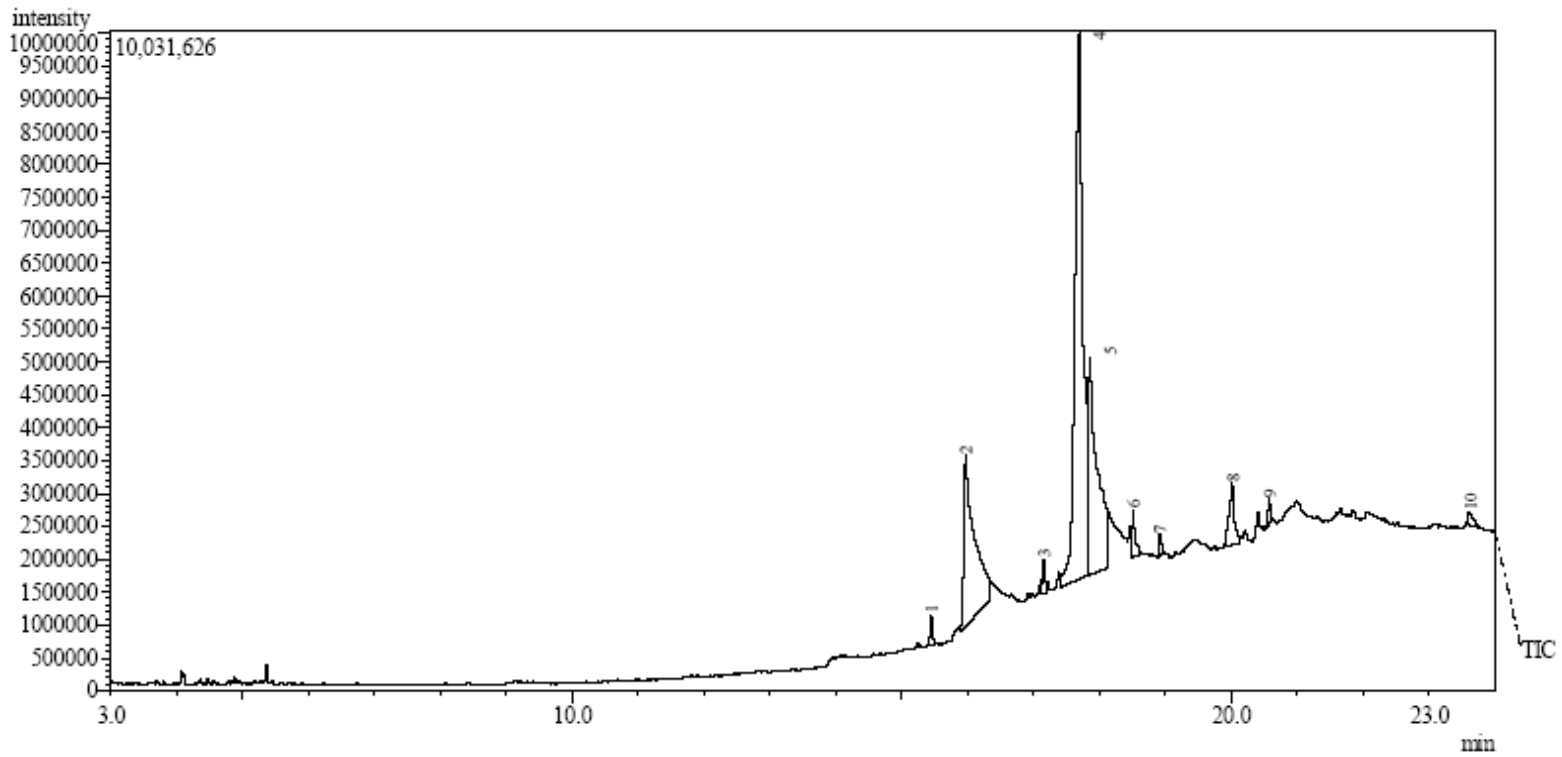

Fig. 2: GC-MS Chromatogram of BSO

Table 4: Fatty Acid Composition of MSO and BSO

\begin{tabular}{|c|c|c|c|c|c|c|c|c|c|}
\hline \multirow[t]{2}{*}{ Peaks } & \multicolumn{2}{|c|}{$\begin{array}{l}\text { Retention Time } \\
\text { (min) }\end{array}$} & \multirow[t]{2}{*}{ Compound } & \multicolumn{2}{|c|}{ Chemical Formula } & \multicolumn{2}{|c|}{$\begin{array}{c}\text { Molecular Weight } \\
(\mathrm{g} / \mathrm{mol})\end{array}$} & \multicolumn{2}{|c|}{$\%$ Area } \\
\hline & MSO & $\mathrm{BSO}$ & & MSO & BSO & MSO & BSO & MSO & BSO \\
\hline 1 & 15.442 & 15.450 & Pentadecanoic Acid & $\mathrm{C}_{17} \mathrm{H}_{34} \mathrm{O}_{2}$ & $\mathrm{C}_{17} \mathrm{H}_{34} \mathrm{O}_{2}$ & 270 & 270 & 0.76 & 0.83 \\
\hline 2 & 16.001 & 15.971 & Palmitic Acid & $\mathrm{C}_{16} \mathrm{H}_{32} \mathrm{O}_{2}$ & $\mathrm{C}_{16} \mathrm{H}_{32} \mathrm{O}_{2}$ & 256 & 256 & 21.99 & 20.02 \\
\hline 3 & 17.154 & 17.159 & Elaidic Acid & $\mathrm{C}_{19} \mathrm{H}_{36} \mathrm{O}_{2}$ & $\mathrm{C}_{19} \mathrm{H}_{36} \mathrm{O}_{2}$ & 296 & 296 & 1.71 & 0.87 \\
\hline 4 & 17.747 & 17.697 & Oleic Acid & $\mathrm{C}_{18} \mathrm{H}_{34} \mathrm{O}_{2}$ & $\mathrm{C}_{18} \mathrm{H}_{34} \mathrm{O}_{2}$ & 282 & 282 & 54.08 & 48.11 \\
\hline 5 & 17.899 & 17.860 & Stearic Acid & $\mathrm{C}_{18} \mathrm{H}_{36} \mathrm{O}_{2}$ & $\mathrm{C}_{18} \mathrm{H}_{36} \mathrm{O}_{2}$ & 284 & 284 & 14.93 & 22.43 \\
\hline 6 & 18.515 & 18.514 & 1-Nonadecanol & $\mathrm{C}_{19} \mathrm{H}_{40} \mathrm{O}$ & $\mathrm{C}_{9} \mathrm{H}_{16} \mathrm{BrNO}$ & 284 & 233 & 0.63 & 1.75 \\
\hline 7 & 20.017 & 18.915 & Linolenic Acid & $\mathrm{C}_{20} \mathrm{H}_{34} \mathrm{O}_{2}$ & $\mathrm{C}_{12} \mathrm{H}_{26} \mathrm{O}$ & 306 & 186 & 0.76 & 0.66 \\
\hline 8 & 20.388 & 20.019 & 5-Cholestene-3ol & $\mathrm{C}_{28} \mathrm{H}_{48} \mathrm{O}$ & $\mathrm{C}_{14} \mathrm{H}_{26} \mathrm{O}$ & 400 & 210 & 2.49 & 3.78 \\
\hline 9 & 20.576 & 20.580 & $\begin{array}{l}\text { 13-Docosenoic Acid methyl } \\
\text { ether }\end{array}$ & $\mathrm{C}_{23} \mathrm{H}_{44} \mathrm{O}_{2}$ & $\mathrm{C}_{23} \mathrm{H}_{44} \mathrm{O}_{2}$ & 352 & 352 & 1.51 & 0.77 \\
\hline 10 & 23.609 & 23.616 & Squalene & $\mathrm{C}_{30} \mathrm{H}_{50}$ & $\mathrm{C}_{30} \mathrm{H}_{50}$ & 410 & 410 & $\begin{array}{l}1.13 \\
100\end{array}$ & $\begin{array}{l}0.78 \\
100\end{array}$ \\
\hline
\end{tabular}




\subsection{Characterization of Cutting Fluids}

The characteristics of the formulated and commercially available (CMO) oil-in-water emulsion cutting fluids used in this study are shown in Table 5.

Table 5: Characteristics of cutting fluids

\begin{tabular}{|c|c|c|c|c|}
\hline$S / N$ & Property & $\begin{array}{l}\text { Melon Seed } \\
\text { (MSO) }\end{array}$ & $\begin{array}{l}\text { Beniseed } \\
\text { (BSO) }\end{array}$ & $\begin{array}{l}\text { Mineral oil } \\
(\mathrm{CMO})\end{array}$ \\
\hline 1 & $\begin{array}{l}\text { Viscosity (n) } \\
\left(\mathrm{mm}^{2} / \mathrm{s}\right)\end{array}$ & 1.53 & 0.86 & 1.00 \\
\hline 2 & $\mathrm{pH}$ Value & 8.2 & 8.7 & 8.9 \\
\hline 3 & Corrosion & Corrosion & Corrosion & Corrosion \\
\hline 3 & Level & Resistant & Resistant & Resistant \\
\hline 4 & Stability & Stable & Stable & Stable \\
\hline 5 & Colour & Milky & Milky & Milky \\
\hline
\end{tabular}

The results shown in Table 5 indicates that the MSO, BSO and CMO are stable, milky and non-corrosive cutting fluids with viscosity of $1.53,0.86$ and 1 $\left(\mathrm{mm}^{2} / \mathrm{s}\right)$ respectively, thereby making it effective and safe-to use

\subsection{Experimental Results and S/N Ratios}

The results of the experiment carried out using the experimental design layout along with their respective signal-to noise $(\mathrm{S} / \mathrm{N})$ ratio values are shown in Table 6 . It can be observed that the value of responses (temperature and surface roughness) changes with variation in the process parameters.

Signal-to noise $(\mathrm{S} / \mathrm{N})$ ratios of individual responses were calculated using Equ. 1 and 2.

Smaller-the better:

$$
\mathrm{S} / \mathrm{N}=-10 \log \frac{1}{n}\left(\sum_{i-1}^{n} y_{i}^{2}\right)
$$

Larger-the better:

$$
\mathrm{S} / \mathrm{N}=-10 \log \frac{1}{n}\left(\sum_{i-1}^{n} \frac{1}{y_{i}^{2}}\right)
$$

where $\mathrm{S} / \mathrm{N}$ is signal to noise ratio, $\mathrm{n}$ is the number of repetition in a trial and $y$ is the measure quality characteristic for the ith repetition

\begin{tabular}{|c|c|c|c|c|c|c|c|c|}
\hline $\begin{array}{l}\text { Run } \\
\text { Order }\end{array}$ & $\begin{array}{l}\text { Cutting } \\
\text { Speed CS } \\
\text { (rev/min) }\end{array}$ & $\begin{array}{c}\text { Feed Rate } \\
\text { FR } \\
(\mathrm{mm} / \mathrm{rev})\end{array}$ & $\begin{array}{l}\text { Depth of } \\
\text { Cut DOC } \\
(\mathrm{mm})\end{array}$ & $\begin{array}{c}\text { Type of } \\
\text { Cutting Fluids } \\
\text { (TCF) (n) } \\
\left(\mathrm{mm}^{2} / \mathrm{s}\right)\end{array}$ & $\begin{array}{c}\text { Surface } \\
\text { Roughness Ra } \\
(\mu \mathrm{m})\end{array}$ & $\begin{array}{l}\text { S/N ratio for } \\
\text { Surface } \\
\text { Roughness }(\mathrm{db})\end{array}$ & $\begin{array}{c}\text { Temperature } \\
\mathrm{T}\left({ }^{\circ} \mathrm{C}\right)\end{array}$ & $\begin{array}{l}\text { S/N ratio for } \\
\text { Temperature } \\
\text { (db) }\end{array}$ \\
\hline 1 & 93 & 0.5 & 0.8 & 1.53 & 1.10 & -0.83 & 40.9 & -32.23 \\
\hline 2 & 93 & 0.9 & 1.2 & 1 & 7.76 & -17.80 & 57.2 & -35.15 \\
\hline 3 & 159 & 0.9 & 0.8 & 1.53 & 9.49 & -19.55 & 59.7 & -35.52 \\
\hline 4 & 159 & 0.7 & 0.8 & 0.86 & 3.44 & -10.73 & 50.1 & -34.00 \\
\hline 5 & 159 & 0.9 & 1 & 1 & 10.13 & -20.11 & 67.1 & -36.53 \\
\hline 6 & 126 & 0.9 & 1.2 & 1 & 8.84 & -18.93 & 60.5 & -35.64 \\
\hline 7 & 126 & 0.7 & 0.8 & 1 & 1.83 & -5.25 & 50.3 & -34.03 \\
\hline 8 & 126 & 0.9 & 0.8 & 1 & 6.67 & -16.48 & 52.4 & -34.39 \\
\hline 9 & 126 & 0.7 & 0.8 & 1.53 & 3.52 & -10.93 & 39.7 & -31.98 \\
\hline 10 & 159 & 0.5 & 1.2 & 1 & 2.53 & -8.06 & 54.8 & -34.78 \\
\hline 11 & 159 & 0.5 & 1 & 1 & 1.97 & -5.89 & 51.6 & -34.25 \\
\hline 12 & 159 & 0.5 & 0.8 & 0.86 & 2.56 & -8.16 & 43.4 & -32.75 \\
\hline 13 & 93 & 0.9 & 1 & 1.53 & 8.74 & -18.83 & 50.5 & -34.07 \\
\hline 14 & 126 & 0.7 & 1.2 & 1 & 1.92 & -5.67 & 61.1 & -35.72 \\
\hline 15 & 93 & 0.7 & 0.8 & 1.53 & 2.86 & -9.13 & 44.6 & -32.99 \\
\hline 16 & 93 & 0.7 & 1.2 & 0.86 & 6.42 & -16.15 & 56.4 & -35.03 \\
\hline 17 & 93 & 0.7 & 1.2 & 1.53 & 2.47 & -7.85 & 44.3 & -32.93 \\
\hline 18 & 93 & 0.5 & 1.2 & 0.86 & 2.03 & -6.15 & 56.9 & -35.10 \\
\hline 19 & 93 & 0.5 & 0.8 & 0.86 & 1.97 & -5.89 & 50.6 & -34.08 \\
\hline 20 & 126 & 0.9 & 0.8 & 1.53 & 8.90 & -18.99 & 41.6 & -32.38 \\
\hline 21 & 159 & 0.7 & 1.2 & 0.86 & 2.23 & -6.97 & 52.5 & -34.40 \\
\hline 22 & 159 & 0.9 & 1 & 1.53 & 8.17 & -18.24 & 80.2 & -38.08 \\
\hline 23 & 126 & 0.9 & 1 & 1.53 & 8.89 & -18.98 & 41.0 & -32.26 \\
\hline 24 & 93 & 0.7 & 1.2 & 1 & 2.96 & -9.43 & 67.7 & -36.61 \\
\hline 25 & 159 & 0.9 & 1.2 & 0.86 & 7.74 & -17.77 & 58.7 & -35.37 \\
\hline 26 & 93 & 0.7 & 0.8 & 1 & 2.34 & -7.38 & 58.2 & -35.30 \\
\hline 27 & 159 & 0.9 & 1.2 & 1.53 & 9.05 & -19.13 & 87.0 & -38.79 \\
\hline 28 & 126 & 0.5 & 1.2 & 0.86 & 1.55 & -3.81 & 55.7 & -34.92 \\
\hline 29 & 159 & 0.7 & 1 & 1.53 & 2.71 & -8.66 & 60.3 & -35.61 \\
\hline 30 & 159 & 0.9 & 0.8 & 0.86 & 7.35 & -17.33 & 48.0 & -33.62 \\
\hline 31 & 126 & 0.5 & 1.2 & 1.53 & 3.31 & -10.40 & 46.1 & -33.27 \\
\hline 32 & 159 & 0.7 & 1.2 & 1 & 3.15 & -9.97 & 61.2 & -35.74 \\
\hline 33 & 159 & 0.7 & 1 & 1 & 2.70 & -8.63 & 59.1 & -35.43 \\
\hline 34 & 93 & 0.9 & 1 & 1 & 8.67 & -18.76 & 53.3 & -34.53 \\
\hline 35 & 126 & 0.5 & 1 & 1.53 & 1.57 & -3.92 & 40.2 & -32.08 \\
\hline
\end{tabular}

Table 6: Experimental process parameters, response values and $S / N$ ratios 


\begin{tabular}{|c|c|c|c|c|c|c|c|c|}
\hline $\begin{array}{l}\text { Run } \\
\text { Order }\end{array}$ & $\begin{array}{l}\text { Cutting } \\
\text { Speed CS } \\
\text { (rev/min) }\end{array}$ & $\begin{array}{c}\text { Feed Rate } \\
\text { FR } \\
(\mathrm{mm} / \mathrm{rev})\end{array}$ & $\begin{array}{l}\text { Depth of } \\
\text { Cut DOC } \\
(\mathrm{mm})\end{array}$ & $\begin{array}{c}\text { Type of } \\
\text { Cutting Fluids } \\
(\mathrm{TCF})(\mathrm{n}) \\
\left(\mathrm{mm}^{2} / \mathrm{s}\right)\end{array}$ & $\begin{array}{c}\text { Surface } \\
\text { Roughness Ra } \\
\qquad(\mu \mathrm{m})\end{array}$ & $\begin{array}{l}\text { S/N ratio for } \\
\text { Surface } \\
\text { Roughness }(d b)\end{array}$ & $\begin{array}{c}\text { Temperature } \\
\mathrm{T}\left({ }^{\circ} \mathrm{C}\right)\end{array}$ & $\begin{array}{c}\text { S/N ratio for } \\
\text { Temperature } \\
\text { (db) }\end{array}$ \\
\hline 36 & 126 & 0.7 & 1 & 1.53 & 1.98 & -5.93 & 43.8 & -32.83 \\
\hline 37 & 159 & 0.5 & 0.8 & 1 & 1.58 & -3.97 & 48.9 & -33.79 \\
\hline 38 & 159 & 0.9 & 1.2 & 1 & 10.01 & -20.01 & 73.1 & -37.28 \\
\hline 39 & 93 & 0.9 & 0.8 & 1 & 8.41 & -18.50 & 69.2 & -36.80 \\
\hline 40 & 159 & 0.5 & 1 & 0.86 & 2.11 & -6.49 & 45.9 & -33.24 \\
\hline 41 & 126 & 0.7 & 0.8 & 0.86 & 2.84 & -9.07 & 48.8 & -33.77 \\
\hline 42 & 126 & 0.9 & 1.2 & 1.53 & 9.69 & -19.73 & 47.8 & -33.59 \\
\hline 43 & 93 & 0.7 & 1 & 0.86 & 5.85 & -15.34 & 53.2 & -34.52 \\
\hline 44 & 93 & 0.9 & 0.8 & 0.86 & 8.56 & -18.65 & 49.7 & -33.93 \\
\hline 45 & 93 & 0.9 & 1.2 & 0.86 & 8.65 & -18.74 & 58.1 & -35.28 \\
\hline 46 & 159 & 0.7 & 0.8 & 1 & 2.35 & -7.42 & 55.2 & -34.84 \\
\hline 47 & 126 & 0.5 & 1.2 & 1 & 2.76 & -8.82 & 70.0 & -36.90 \\
\hline 48 & 159 & 0.5 & 1.2 & 0.86 & 1.54 & -3.75 & 45.1 & -33.08 \\
\hline 49 & 93 & 0.5 & 1 & 0.86 & 2.09 & -6.40 & 52.4 & -34.39 \\
\hline 50 & 93 & 0.7 & 0.8 & 0.86 & 2.74 & -8.76 & 50.7 & -34.10 \\
\hline 51 & 126 & 0.9 & 1.2 & 0.86 & 7.70 & -17.73 & 54.5 & -34.73 \\
\hline 52 & 126 & 0.9 & 0.8 & 0.86 & 8.39 & -18.48 & 48.0 & -33.62 \\
\hline 53 & 126 & 0.9 & 1 & 0.86 & 9.42 & -19.48 & 50.8 & -34.12 \\
\hline 54 & 159 & 0.7 & 1 & 0.86 & 3.69 & -11.34 & 50.0 & -33.98 \\
\hline 55 & 126 & 0.5 & 1 & 0.86 & 1.64 & -4.30 & 52.5 & -34.40 \\
\hline 56 & 93 & 0.9 & 1 & 0.86 & 8.26 & -18.34 & 54.4 & -34.71 \\
\hline 57 & 159 & 0.9 & 1 & 0.86 & 9.49 & -19.55 & 51.6 & -34.25 \\
\hline 58 & 126 & 0.7 & 1.2 & 1.53 & 3.72 & -11.41 & 45.2 & -33.10 \\
\hline 59 & 93 & 0.5 & 0.8 & 1 & 1.46 & -3.29 & 48.7 & -33.75 \\
\hline 60 & 93 & 0.5 & 1.2 & 1 & 1.62 & -4.19 & 46.8 & -33.40 \\
\hline 61 & 126 & 0.9 & 1 & 1 & 8.13 & -18.20 & 57.2 & -35.15 \\
\hline 62 & 93 & 0.5 & 1 & 1.53 & 1.32 & -2.41 & 41.5 & -32.36 \\
\hline 63 & 126 & 0.7 & 1 & 0.86 & 1.91 & -5.62 & 55.2 & -34.84 \\
\hline 64 & 93 & 0.7 & 1 & 1 & 1.94 & -5.76 & 64.8 & -36.23 \\
\hline 65 & 126 & 0.7 & 1.2 & 0.86 & 2.56 & -8.16 & 60.2 & -35.59 \\
\hline 66 & 93 & 0.7 & 1 & 1.53 & 3.7 & -11.36 & 48.2 & -33.66 \\
\hline 67 & 159 & 0.5 & 1.2 & 1.53 & 1.33 & -2.48 & 37.9 & -31.57 \\
\hline 68 & 159 & 0.7 & 0.8 & 1.53 & 2.25 & -7.04 & 51 & -34.15 \\
\hline 69 & 159 & 0.5 & 1 & 1.53 & 2.49 & -7.92 & 42.6 & -32.59 \\
\hline 70 & 159 & 0.7 & 1.2 & 1.53 & 2.91 & -9.28 & 76.3 & -37.65 \\
\hline 71 & 159 & 0.5 & 0.8 & 1.53 & 1.22 & -1.73 & 40.7 & -32.19 \\
\hline 72 & 126 & 0.5 & 1 & 1 & 1.59 & -4.03 & 55.2 & -34.84 \\
\hline 73 & 126 & 0.5 & 0.8 & 1.53 & 1.49 & -3.46 & 40.1 & -32.06 \\
\hline 74 & 126 & 0.5 & 0.8 & 1 & 1.66 & -4.40 & 50.3 & -34.03 \\
\hline 75 & 126 & 0.7 & 1 & 1 & 3 & -9.54 & 54.4 & -34.71 \\
\hline 76 & 126 & 0.5 & 0.8 & 0.86 & 1.86 & -5.39 & 50.6 & -34.08 \\
\hline 77 & 159 & 0.9 & 0.8 & 1 & 9.84 & -19.86 & 62 & -35.85 \\
\hline 78 & 93 & 0.9 & 1.2 & 1.53 & 9.5 & -19.55 & 55.6 & -34.90 \\
\hline 79 & 93 & 0.9 & 0.8 & 1.53 & 8.75 & -18.84 & 44 & -32.87 \\
\hline 80 & 93 & 0.5 & 1.2 & 1.53 & 1.74 & -4.81 & 42.2 & -32.51 \\
\hline 81 & 93 & 0.5 & 1 & 1 & 1.7 & -4.61 & 46.3 & -33.31 \\
\hline
\end{tabular}

\subsection{Analysis of Experimental Results}

\subsubsection{Analysis of Variance (ANOVA)}

ANOVA was conducted to study the significant effects of experimental factors. This analysis was conducted using confidence level of $95 \%$ at significant level of 0.05 ( $5 \%$ ). Table 7 and 8 shows the degree of freedom (DOF), sum of square (SS), mean square values (MS), $f$-value and percentage contribution $(p)$ for surface roughness and cutting temperature $(T)$. The ANOVA for surface roughness shown in Table 7 shows a percentage error of 6.53 with feed rate $(92.93$ $\%$ ) indicating the most significant parameter, followed by depth of cut $(0.28 \%)$ and cutting speed $(0.13 \%)$. Finally, the least significant factor is type of cutting fluids $(0.12 \%)$.

Table 7: ANOVA for surface roughness

\begin{tabular}{lccccc}
\hline Factors & DOF & SS & MS & F & P $(\%)$ \\
\hline CS $($ rev/min) & 2 & 1.04 & 0.52 & 0.727 & 0.13 \\
FR (mm/rev) & 2 & 733.07 & 366.6 & 512.76 & 92.93 \\
DOC $(\mathrm{mm})$ & 2 & 2.218 & 1.109 & 1.5514 & 0.28 \\
TCF $\left(\mathrm{mm}^{2} / \mathrm{s}\right)$ & 2 & 1.007 & 0.504 & 0.7044 & 0.13 \\
Error & 74 & 51.468 & 0.715 & & 6.53 \\
Total & 80 & 788.80 & 9.860 & & 100 \\
\hline
\end{tabular}


Table 8: ANOVA for cutting temperature

\begin{tabular}{lccccc}
\hline Factors & DOF & SS & MS & F & P $(\%)$ \\
\hline CS $(\mathrm{rev} / \mathrm{min})$ & 2 & 1301.3 & 650.65 & 110.10 & 18.08 \\
FR $(\mathrm{mm} / \mathrm{rev})$ & 2 & 1980 & 990 & 167.5295 & 27.51 \\
DOC $(\mathrm{mm})$ & 2 & 1611.2 & 805.6 & 136.3251 & 22.39 \\
TCF $\left(\mathrm{mm}^{2} / \mathrm{s}\right)$ & 2 & 1867 & 933.5 & 157.9685 & 25.94 \\
Error & 74 & 437.23 & 5.909 & & 6.08 \\
Total & 80 & 7196.8 & 89.96 & & 100 \\
\hline
\end{tabular}

Also, the ANOVA for cutting temperature shown in Table 8 shows a percentage error of 6.08 while feed rate $(27.51 \%)$ specifies the most significant parameter, followed by type of cutting fluid $(25.94 \%)$, depth of cut (22.39\%) and the least significant factor, type of cutting speed (18.08\%). The effects of all the factors are significant since their individual $p$-values are greater than $0.05 \%$.

\subsubsection{Empirical Model Equations}

The empirical model equation for surface roughness and cutting temperature along with their respective Rsq values are shown in Equation 3 and 4.

$\mathrm{Ra}(\mu \mathrm{m})=-7.76+0.00024 \mathrm{CS}+17.2 \mathrm{FR}$

$$
+0.95 \mathrm{DOC}+0.034 \mathrm{TCF}
$$

Rsq $=80.95 \%$ and Rsq (adj) $=79.95 \%$

$\mathrm{T}(\mathrm{oC})=20.13+0.0604 \mathrm{CS}$

$$
-21.79 \text { FR+ 18.1 DOC+7.15 TCF }
$$

Rsq $=61.85 \%$ and Rsq (adj) $=58.27 \%$

The Rsq for cutting temperature shown in Equ. 4 is less than $80 \%$. This may be due to noise which result from experimental uncertainty.

\subsubsection{D Surface Plots}

The 3-D surface plot of surface roughness and cutting temperature are shown in Fig. 3 and 4. Fig. 3 and 4 shows how change in cutting speed and feed rate affects the surface roughness and cutting temperature when the depth of cut and cutting fluid are kept constant at $1 \mathrm{~mm}$ and $1.195 \mathrm{~mm}^{2} / \mathrm{s}$ respectively. The plots also indicate that as cutting speed increases, feed rate also increases and vice versa.

\subsection{Grey Relational Analysis (GRA)}

As specified by [13], GRA optimization procedure involves calculating the grey relational generation (GRG) of individual responses using the $\mathrm{S} / \mathrm{N}$ ratios values shown in Table 6. GRG was calculated using smaller-the-better attributes $\left(x_{i j}\right)$ as shown in Equ. 5. This is followed by the conversion of GRG to grey relational coefficient (GRC) using Equ. 6. The final stage of GRA was the calculation of grey relational grade using Equ. 7. The results of GRA are shown in Table 9.

Smaller-the better,

$$
\left(\mathrm{x}_{\mathrm{ij}}\right)=\frac{\bar{y}_{i j}-y_{i j}}{\overline{y_{j}}-\underline{y}_{j}}
$$

$(i=1,2,3 \ldots . m$ and $j=1,2,3 \ldots n)$

Where, $y_{i}=\left(y_{i 1}, y_{i 2}, \ldots, y_{i j}, \ldots, y_{i n}\right), y_{i j}$ is the performance value of attribute $j$ of alternative $i$ and $\overline{y_{j}}=\max \left\{\mathrm{y}_{\mathrm{ij}}, \mathrm{i}=1,2, \ldots, \mathrm{m}\right\}$ and $y_{j}=\min \left\{\mathrm{y}_{\mathrm{ij}}, \mathrm{i}\right.$ $=1,2, \ldots, \mathrm{m}\}$.

\section{Surface Plot of Surface Roughness Ra $(\mu \mathrm{m})$}

$\begin{array}{lr}\text { Hold Values } \\ \begin{array}{|r}\hline \multicolumn{1}{|c}{} \\ \text { Depth of Cut }(\mathrm{mm})\end{array} \\ \text { Type of Cutting Fluids (mm2/s) } & 1.195\end{array}$

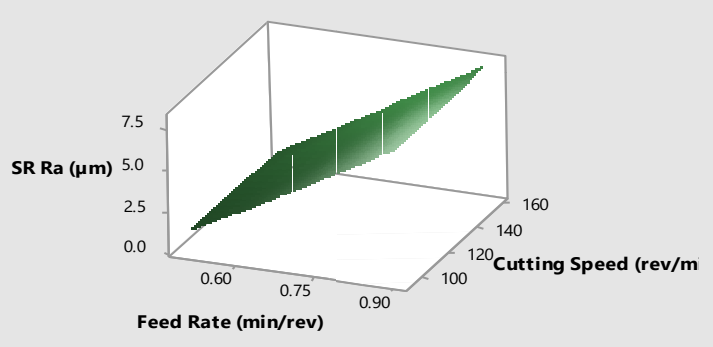

Fig. 3. 3D Surface plot for Surface Roughness

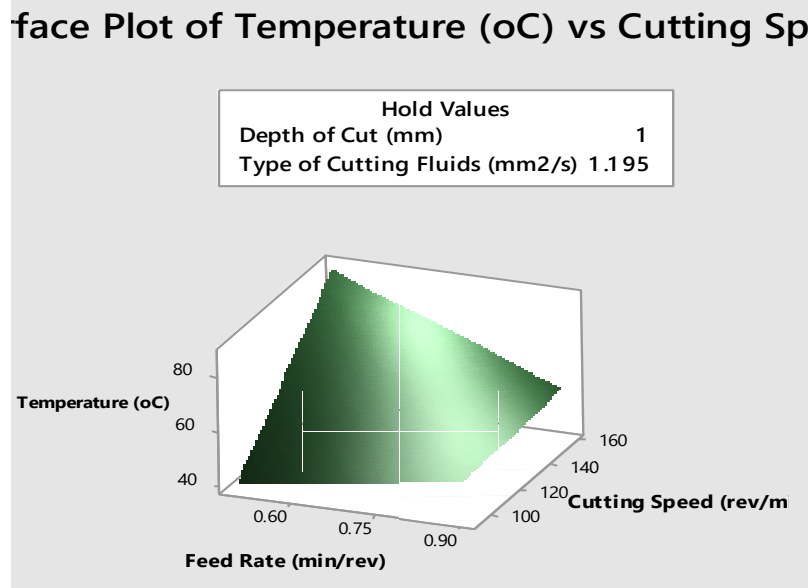

Fig. 4. 3D Surface plot for Temperature

$$
\mathrm{GRC}, \gamma\left(\mathrm{x}_{0 \mathrm{j},} \mathrm{x}_{\mathrm{ij}}\right)=\frac{\Delta_{\text {min }}+\beta \Delta_{\text {max }}}{\Delta_{i j}+\beta \Delta_{\max }}
$$

$(\mathrm{j}=1,2, \ldots, \mathrm{n}$ and $\mathrm{i}=1,2, \ldots, \mathrm{m})$

$\Delta_{i j}=x_{0 j}-x_{i j}, \Delta_{\min }=\min \left(\Delta_{i j}, \mathrm{i}=1,2, \ldots, \mathrm{m} ; \mathrm{j}=1,2\right.$, $\ldots, \mathrm{n})$,

$\Delta_{\max }=\max \left(\Delta_{\mathrm{ij},} \mathrm{i}=1,2, \ldots, m ; j=1,2, \ldots, n\right)$ and $\beta$ is the distinguishing coefficient, $\beta \in[0,1]$. The 
aim of the distinguishing coefficient was to compress or expand the range of the grey relational coefficient and 0.5 is the widely accepted value [19].

$$
\text { Grade, } \varphi\left(x_{0}, x_{i}\right)=\sum_{j=1}^{n} \mathrm{w}_{\mathrm{j}} \beta\left(x_{o j}, x_{i j}\right)
$$

$(\mathrm{i}=1,2,3 \ldots \ldots \mathrm{m}) ; \quad w j=$ weight of attribute $j$. $\sum_{j=1}^{n} \mathrm{w}_{\mathrm{j}}=1[18]$

The resulting factor effects of the process factors obtained using the grey relational grades as shown in Tables 9. The main effect plots obtained is as shown in Fig. 5.

Table 9: Results of Grey Relational Analysis

\begin{tabular}{|c|c|c|c|c|c|}
\hline \multirow{2}{*}{ כ্ত্ৰ } & \multicolumn{2}{|c|}{ Grey Relational Generation } & \multicolumn{2}{|c|}{ Grey Relational Coefficient } & \multirow{2}{*}{ Grade } \\
\hline & $\mathrm{Ra}$ & $\mathrm{T}\left({ }^{\circ} \mathrm{C}\right)$ & $\mathrm{Ra}$ & $\mathrm{T}\left({ }^{\circ} \mathrm{C}\right)$ & \\
\hline 1 & 1 & 1 & 0.333 & 0.340 & 0.337 \\
\hline 2 & 0 & 0.03 & 0.806 & 0.375 & 0.591 \\
\hline 3 & 0.88 & 0.17 & 0.945 & 0.380 & 0.663 \\
\hline 4 & 0.97 & 0.18 & 0.507 & 0.361 & 0.434 \\
\hline 5 & 0.51 & 0.11 & 1.000 & 0.394 & 0.697 \\
\hline 6 & 1 & 0.23 & 0.891 & 0.382 & 0.636 \\
\hline 7 & 0.94 & 0.19 & 0.393 & 0.361 & 0.377 \\
\hline 8 & 0.23 & 0.12 & 0.727 & 0.365 & 0.546 \\
\hline 9 & 0.81 & 0.13 & 0.512 & 0.338 & 0.425 \\
\hline 10 & 0.52 & 0.02 & 0.444 & 0.370 & 0.407 \\
\hline 11 & 0.38 & 0.15 & 0.404 & 0.364 & 0.384 \\
\hline 12 & 0.26 & 0.13 & 0.446 & 0.346 & 0.396 \\
\hline 13 & 0.38 & 0.06 & 0.883 & 0.362 & 0.622 \\
\hline 14 & 0.93 & 0.12 & 0.400 & 0.383 & 0.392 \\
\hline 15 & 0.25 & 0.19 & 0.467 & 0.349 & 0.408 \\
\hline 16 & 0.43 & 0.07 & 0.709 & 0.374 & 0.541 \\
\hline 17 & 0.79 & 0.16 & 0.440 & 0.348 & 0.394 \\
\hline 18 & 0.36 & 0.06 & 0.408 & 0.375 & 0.392 \\
\hline 19 & 0.28 & 0.17 & 0.404 & 0.362 & 0.383 \\
\hline 20 & 0.26 & 0.12 & 0.896 & 0.342 & 0.619 \\
\hline 21 & 0.94 & 0.04 & 0.423 & 0.366 & 0.394 \\
\hline 22 & 0.32 & 0.13 & 0.838 & 0.418 & 0.628 \\
\hline 23 & 0.9 & 0.3 & 0.894 & 0.341 & 0.618 \\
\hline 24 & 0.94 & 0.03 & 0.474 & 0.396 & 0.435 \\
\hline 25 & 0.45 & 0.24 & 0.805 & 0.378 & 0.592 \\
\hline 26 & 0.88 & 0.18 & 0.431 & 0.377 & 0.404 \\
\hline 27 & 0.34 & 0.17 & 0.907 & 0.430 & 0.669 \\
\hline 28 & 0.95 & 0.34 & 0.371 & 0.372 & 0.372 \\
\hline 29 & 0.15 & 0.16 & 0.457 & 0.381 & 0.419 \\
\hline 30 & 0.41 & 0.19 & 0.776 & 0.356 & 0.566 \\
\hline 31 & 0.86 & 0.1 & 0.498 & 0.352 & 0.425 \\
\hline 32 & 0.5 & 0.08 & 0.487 & 0.383 & 0.435 \\
\hline 33 & 0.47 & 0.19 & 0.456 & 0.379 & 0.418 \\
\hline 34 & 0.4 & 0.18 & 0.877 & 0.367 & 0.622 \\
\hline 35 & 0.93 & 0.14 & 0.373 & 0.339 & 0.356 \\
\hline 36 & 0.16 & 0.02 & 0.405 & 0.347 & 0.376 \\
\hline 37 & 0.26 & 0.06 & 0.374 & 0.358 & 0.366 \\
\hline 38 & 0.16 & 0.1 & 0.990 & 0.406 & 0.698 \\
\hline
\end{tabular}




\begin{tabular}{|c|c|c|c|c|c|}
\hline \multirow{2}{*}{ 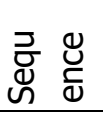 } & \multicolumn{2}{|c|}{ Grey Relational Generation } & \multicolumn{2}{|c|}{ Grey Relational Coefficient } & \multirow{2}{*}{ Grade } \\
\hline & $\mathrm{Ra}$ & $\mathrm{T}\left({ }^{\circ} \mathrm{C}\right)$ & $\mathrm{Ra}$ & $\mathrm{T}\left({ }^{\circ} \mathrm{C}\right)$ & \\
\hline 39 & 0.99 & 0.27 & 0.856 & 0.398 & 0.627 \\
\hline 40 & 0.92 & 0.24 & 0.414 & 0.352 & 0.383 \\
\hline 41 & 0.29 & 0.08 & 0.466 & 0.358 & 0.412 \\
\hline 42 & 0.43 & 0.1 & 0.962 & 0.356 & 0.659 \\
\hline 43 & 0.98 & 0.09 & 0.669 & 0.367 & 0.518 \\
\hline 44 & 0.75 & 0.14 & 0.868 & 0.360 & 0.614 \\
\hline 45 & 0.92 & 0.11 & 0.876 & 0.377 & 0.626 \\
\hline 46 & 0.93 & 0.17 & 0.432 & 0.371 & 0.401 \\
\hline 47 & 0.34 & 0.15 & 0.460 & 0.400 & 0.430 \\
\hline 48 & 0.41 & 0.25 & 0.371 & 0.350 & 0.360 \\
\hline 49 & 0.15 & 0.07 & 0.413 & 0.365 & 0.389 \\
\hline 50 & 0.29 & 0.13 & 0.459 & 0.362 & 0.410 \\
\hline 51 & 0.41 & 0.12 & 0.801 & 0.370 & 0.586 \\
\hline 52 & 0.88 & 0.15 & 0.855 & 0.356 & 0.605 \\
\hline 53 & 0.92 & 0.1 & 0.938 & 0.362 & 0.650 \\
\hline 54 & 0.97 & 0.12 & 0.524 & 0.360 & 0.442 \\
\hline 55 & 0.55 & 0.11 & 0.379 & 0.366 & 0.372 \\
\hline 56 & 0.18 & 0.13 & 0.845 & 0.370 & 0.607 \\
\hline 57 & 0.91 & 0.15 & 0.945 & 0.364 & 0.655 \\
\hline 58 & 0.97 & 0.13 & 0.526 & 0.350 & 0.438 \\
\hline 59 & 0.55 & 0.07 & 0.364 & 0.358 & 0.361 \\
\hline 60 & 0.13 & 0.1 & 0.377 & 0.354 & 0.365 \\
\hline 61 & 0.17 & 0.09 & 0.835 & 0.375 & 0.605 \\
\hline 62 & 0.9 & 0.17 & 0.353 & 0.342 & 0.347 \\
\hline 63 & 0.08 & 0.04 & 0.400 & 0.371 & 0.385 \\
\hline 64 & 0.25 & 0.15 & 0.402 & 0.390 & 0.396 \\
\hline 65 & 0.26 & 0.22 & 0.446 & 0.381 & 0.414 \\
\hline 66 & 0.38 & 0.19 & 0.524 & 0.357 & 0.440 \\
\hline 67 & 0.55 & 0.1 & 0.354 & 0.333 & 0.343 \\
\hline 68 & 0.09 & 0 & 0.424 & 0.363 & 0.394 \\
\hline 69 & 0.32 & 0.12 & 0.442 & 1.000 & 0.721 \\
\hline 70 & 0.37 & 1 & 0.471 & 0.412 & 0.441 \\
\hline 71 & 0.44 & 0.28 & 0.344 & 0.340 & 0.342 \\
\hline 72 & 0.05 & 0.03 & 0.375 & 0.371 & 0.373 \\
\hline 73 & 0.17 & 0.15 & 0.367 & 0.339 & 0.353 \\
\hline 74 & 0.14 & 0.02 & 0.380 & 0.361 & 0.371 \\
\hline 75 & 0.19 & 0.12 & 0.477 & 0.370 & 0.423 \\
\hline 76 & 0.45 & 0.15 & 0.396 & 0.362 & 0.379 \\
\hline 77 & 0.24 & 0.12 & 0.975 & 0.385 & 0.680 \\
\hline 78 & 0.99 & 0.2 & 0.945 & 0.372 & 0.659 \\
\hline 79 & 0.97 & 0.16 & 0.883 & 0.347 & 0.615 \\
\hline 80 & 0.93 & 0.06 & 0.387 & 0.343 & 0.365 \\
\hline 81 & 0.21 & 0.04 & 0.383 & 0.352 & 0.368 \\
\hline
\end{tabular}



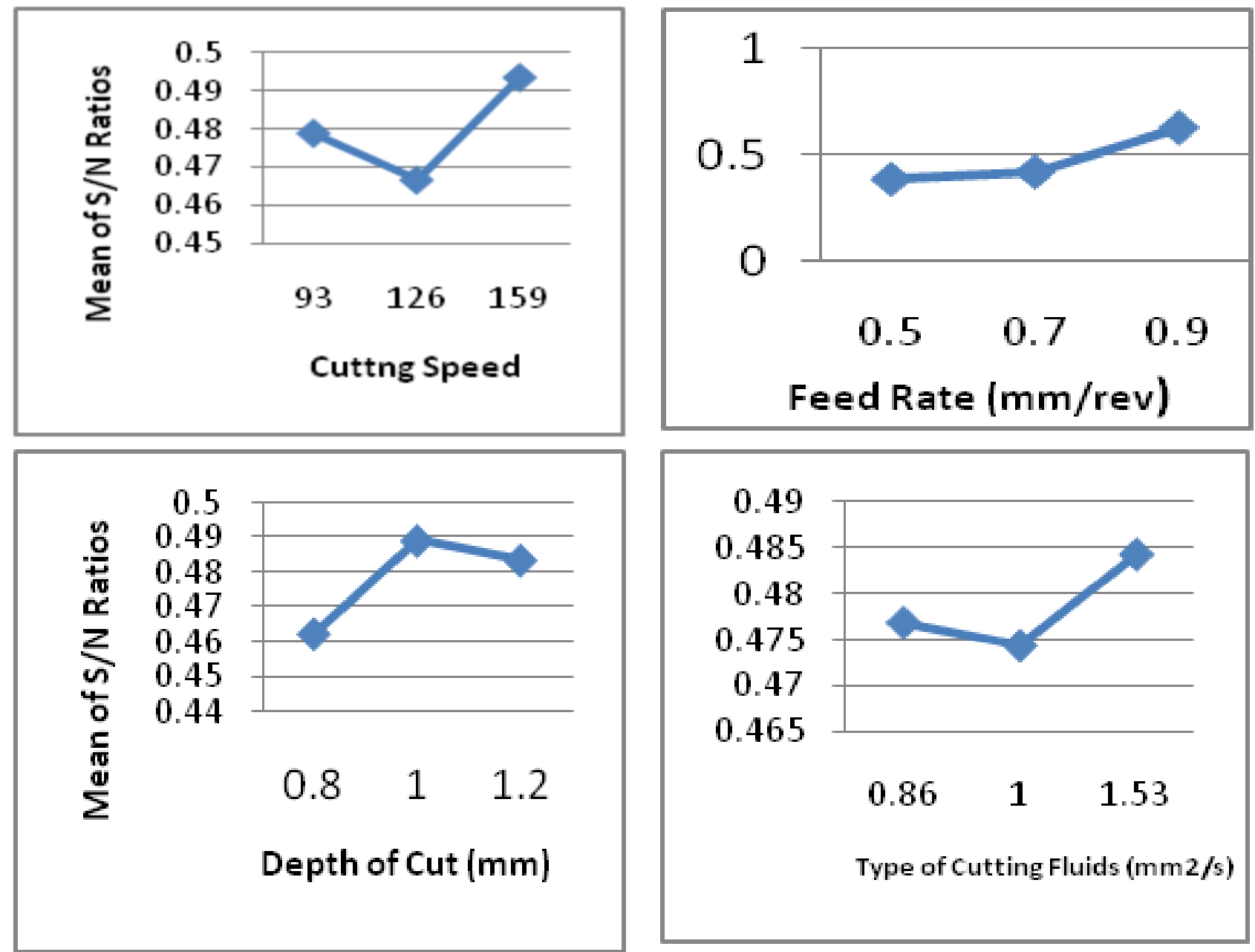

Figure 5. Plots of Factor Effects

The main effect plots shown in Fig. 5 indicates that optimum multi-response machining performance can be achieved using cutting speed of $159 \mathrm{~m} / \mathrm{s}$, feed rate of $0.9 \mathrm{~mm} / \mathrm{rev}$, cutting depth of $1 \mathrm{~mm}$ as well as a commercially available mineral oil of $1.53 \mathrm{~mm}^{2} / \mathrm{s}$ viscosity. Any change in these optimal parameters may lead to poor performance of the turning process.

\section{CONCLUSIONS}

The properties of melon seed and beniseed oils were investigated and the performance evaluation of these oil-based cutting fluids involving the formulation of beniseed and melon seed oil cutting fluids have been carried out. The formulated oil cutting fluids were used in turning AISI 304L alloy steel using a tungsten carbide cutting tool and compared with commercial mineral oil- based cutting fluid. The performance evaluation of formulated cutting fluids was investigated by determining its surface roughness and cutting temperature. The following conclusions were drawn: i. The viscosities of the melon seed oil and beniseed oil based cutting fluids were $1.53 \mathrm{~mm}^{2 / \mathrm{s}}$ and $0.86 \mathrm{~mm}^{2} / \mathrm{s}$, while their $\mathrm{pH}$ values were 8.2 and 8.7 respectively.

ii. The fatty acid composition of the melon seed and beniseed oil influenced the $\mathrm{pH}$ levels and viscosities of their respective cutting fluid, which also affected their performances during machining of AISI 304L alloy steel, using tungsten carbide cutting tool.

iii. The optimal multi-response turning parameters was achieved using cutting speed of 159 $\mathrm{rev} / \mathrm{min}$ (level 3), feed rate of $0.9 \mathrm{~mm} / \mathrm{rev}$ (level 3), depth of cut of $1 \mathrm{~mm}$ (level 2) and type of cutting fluid of $1.53 \mathrm{~mm} / \mathrm{s}$ (level 3 ).

iv. Also, the ANOVA results show that feed rate has most significant effect on the surface roughness and cutting temperature.

\section{REFERENCES}

[1] Sahu, M. K., Verma, M., \& Khandelwal A. K. Experimental Investigation of Machining Parameter in Turning Operation Using Full 
Factorial Design. Journal of Harmonized Research in Engineering. 2(2), 2014. Pp $301-$ 316. ISSN 2347 - 7393.

[2] Kawi, A. A. Temperatures Behaviour of Some Alloy Steel in Turning Process Under Different Operating Conditions. Al-Qadisiya Journal for Engineering Sciences. Volume 4, No. 3 of 2011

[3] Thamizhmanii, S., Sapurudin, S., \& Hasan, S., Analysis of Surface Roughness by Turning Process Using Taguchi Method. Journal of Achievements in Materials and Manufacturing Engineering. Vol. 20, Issues 1-2, JanuaryFebruary, 2007. PP. 503-506

[4] Rajesh, C. Tune - In Optimization Processes of AISI 4140 in Raw Turning Operation Using CVD Coated Insert. International Journal of Advances in Engineering and Technology. July, 2014, Volume 7, Issue 3, pp. 980-990

[5] Weinert, K., Inasaka, I., Sutherland, J. W., \& Wakabayashi, T. Dry Machining and Minimum Quantity Lubrication. CIRP Annals Manufacturing Technology. Volume 53, No.2, 2004, pp 511-537.

[6] Sharif, S., Yusof, N. M., Idris, M. H., Ahmad, Z. A., Sudin, I., Ripin, A., \& Zin, A. H. M.. Feasibility Study of Using Vegetable Oil as a Cutting Lubricant Through The Use of Minimum Quantity Lubrication During Machining. Research Vot No: 78055. Department of Manufacturing and Industrial Engineering, Faculty of Mechanical Engineering, Universiti Teknologi Malaysia., 2009.

[7] Hill, K. Fats and Oils as Oleochemical Raw Materials. Pure and Applied Chemistry. Volume 72. 2000. PP 1255-1264,

[8] Hwang Y.K., \& Lee C.M., Surface Roughness and Cutting Force Prediction in MQL and Wet Turning Process of Process of AISI 1045 Using Design of Experiment. Journal of Mechanical Science and Technology. 24 (8) 2010. pp 1669-1677.

[9] Lawal, S. A., Choudhury, I .A., \& Nukman, Y. Experimental Evaluation and Optimization of Flank Wear During Turning of AISI 4340 Steel with Coated Carbide Inserts Using Different Cutting Fluids. Journal of the Institution of Engineers India: Series C. Mechanical, Production, Aerospace and Marine Engineering. 96, (1), 2014, pp. 21-28.

[10] Bajić, D., \& Majce, I. (2006). Optimisation of Parameters of Turning Process. International
Scientific Conference on Production Engineering. (June-July, 2006). Zagreb Lumbarda, Hrvatska. pp 129-136.

[11] Onuoha, O. J, Suitability of Vegetable Based oils in Orthogonal Machining of AISI 1330 Carbon Steel Using Taguchi Method. Unpublished PhD Thesis submitted to the Mechanical Engineering Department, School of Engineering and Engineering Technology, Federal University of Technology, Minna, Niger State, Nigeria.

[12] Yu, Y., Guo, Y., Wang, L., \& Tang, E. Development of Environmentally Friendly WaterBased Synthetic Metal-Cutting Fluid. Modern Applied Science. Volume 4, No. 1, January, 2010. pp 53-58.

[13] Yiyo, K., Taho,Y.\& Guan, W. H. The use of a greybased Taguchi method for optimizing multiresponse simulation problems. Engineering Optimization, 40(6), 517-52.

[14] Muniz, C. A. S., Dantas, T. N. C., Moura, E.F., Neto, A. A. D., \& Gurgel, A. Novel Formulations of Cutting Fluids using Naphtenic Basic Oil. Brazilian Journal of Petroleum and Gas. Volume 2, No.4, pp. 143-153, 2008.

[15] Alves, S. M., \& Oliveira, J. F. G. Vegetable Based Cutting Fluid - An Environmental Alternative to Grinding Process. $15^{\text {th }}$ CIPR International Conference on Life cycle Engineering, 2008.

[16] Sunday Albert Lawal; Imtiaz Ahmed Choudhury and Yusoff Nukman. Evaluation of vegetable and mineral oil-in-water emulsion cutting fluids in turning AISI 4340 steel with coated carbide tools. Journal of Cleaner Production, 66, 610 - 618.

[17] Godfrey, D., \& Herguth, H. R. Back to Basics; Physical and Chemical Properties of Industrial Mineral Oils Affecting Lubrication. Part 1-5. Society of Tribologists and Lubrication Engineers, Illinois. 1995. Available: http://www.stle.org/ of 17 August, 2016.

[18] Oseni, M. I., Agbi, B. E., and Ogamenyi, I. O. Extraction and Analysis of Chemo-physical Properties of Yellow Oleander Oil as Lubricant. British Journal of Applied Science \& Technology. 4 (6), pp 1020-1029, 2014.

[19] Chin, P. F. Manufacturing process optimization for wear property of fiber-reinforced polybutylene terephthalate composites with grey relational analysis, Wear, 254, 298-306, 2003. 